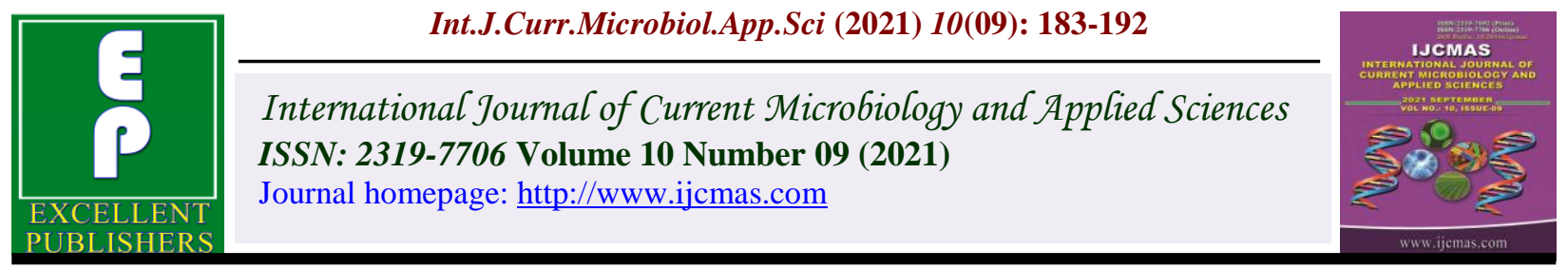

Original Research Article

https://doi.org/10.20546/ijcmas.2021.1009.021

\title{
Estimation of Character Association among Yield Attributing Traits in Mungbean (Vigna radiata (L.) Wilczek)
}

\author{
Gitesh Padol*, G. R. Lavanya and Rohini Kurandale \\ Department of Genetics and Plant Breeding, Naini Agricultural Institute, SHUATS, \\ Prayagraj (U.P.) - 211 007, India \\ *Corresponding author
}

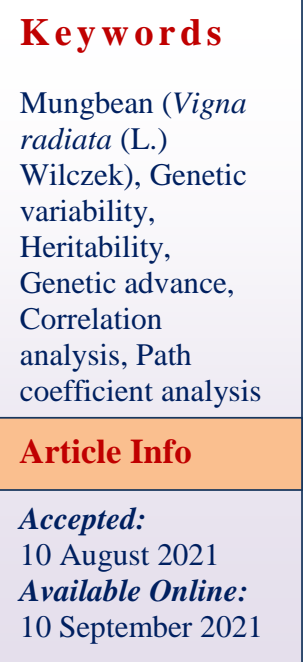

A B S T R A C T

Twenty one mungbean (Vigna radiate L.Wilczek) genotype were evaluated for the estimation of genetic variability, heritability, genetic advance, correlation coefficient, and path coefficient analysis for yield and its component traits. A considerable amount of genetic variability was observed among all the genotypes for all the character under study. GCV and PCV were highest for number of primary branches per plant, followed by 100 seed weight and number of clusters per plant. High genetic advance coupled with high heritability were observed 100 seed weight followed by number of clusters per plant, plant height and number of primary branches per plant. Combined results of correlation coefficient and path analysis revealed that number of primary branches per plant, 100 seed weight, biological yield and harvest index are major component traits for the improvement of grain yield.

\section{Introduction}

Mungbean (Vigna radiata (L.) Wilczek) is an Asiatic species of the pan-tropical genus Vigna with a considerable importance as it is a widely cultivated pulse crop, because of its adaptation to short growth duration, low water requirement, soil fertility and because it can be used in crop rotation practices also. Other properties like easy digestibility and low proportions of flatulence factors also add to its value among the pulse crops. Mungbean is a major source of protein and minerals for the predominantly vegetarian population of India. Hundred grams of mungbean seeds composed of carbohydrates $(62.62 \mathrm{~g})$, sugars $(6.60 \mathrm{~g})$, dietary fibre $(16.3 \mathrm{~g})$, vitamin-C $(4.8 \mathrm{mg})$, magnesium (189 mg), phosphorous (367 mg), potassium $(1246 \mathrm{mg})$ and sodium $(15 \mathrm{mg})$. Average protein content in the seeds is around 23\% (USDA Nutrient Database). India is the largest producer of mungbean, where it is the 
third most important pulse crop followed by pigeonpea and chickpea. Seeds are easily digestible and also very good source of minerals (calcium, iron (4-7mg/100g), zinc $(3 \mathrm{mg} / 100 \mathrm{~g})$, potassium and phosphorus) and vitamins (folate and vitamin $\mathrm{K}$ ) and dietary fibers. Sprouted seeds of mungbean are nutritionally enhanced as ascorbic acid is synthesized with increment in riboflavin and thiamine Mung was sown over an area of 4.26 $\mathrm{M} / \mathrm{ha}$ in (kharif + rabi) and recorded a production of $2.01 \mathrm{M} / \mathrm{t}$ at and yield level of 472 kg/ha. (2017-2018) Rajasthan, MP, Maharashtra, Karnataka, Bihar, AP, Odisha, Tamil Nadu, Gujarat and Telangana have been the major states. (Source: Pulses Revolution From Food to Nutritional Security).

The protein is comparatively rich in lysine, an amino acid predominantly deficit in cereal grains (Baskaran et al., 2009). Pulses are the chief source of protein to meet the nutritional requirement of the masses in the world. Pulses have 20 to $25 \%$ protein by weight, which is twice in comparison to wheat and thrice in comparison to rice. Nutrition scientists emphasize the value of pulses as a source of protein and fiber. Biological nitrogen fixation is an important aspect of sustainable and environment friendly agriculture and role of pulses in this context hardly need any emphasis. Legumes, besides helping in maintaining the soil fertility through biological nitrogen fixation, are major source of protein for human consumption and provide high quality crop can also be grown as intercrop with sugarcane, pigeonpea, poplar and orchards. Thus, there is a great scope of increasing area and production of summer mungbean (Sekhon et al., 2007), however, the productivity of mungbean is still very low. Maximization of yield is major objective in all the crops. Yield is a complex character associated with various contributing characters which are interrelated among themselves. For developing suitable selection strategy the knowledge of genetic variability present in the available germplasm for yield and its associated characters is important.

For any crop improvement programme, selection of superior parents is an essential prerequisite especially for the traits showing higher heritability and genetic advance for various traits (Khan et al., 2005). The information on extent of variability parameters may be helpful to improve the yield by selecting the yield component traits because yield is a complex trait, whose manifestation depends on the component traits. Generally, the estimates of heritability (h2) of traits are environment and population specific (Shimelis and Rhandzu, 2010). These estimates should be incorporated and specifically applied only to the population and environment sampled (Dudleyand Moll, 1969). Thus, selection made on the basis of $\mathrm{h} 2$ alone is likely to be misleading and it becomes necessary to determine the parameters under targeted production environment. Thus, selection of traits based on h2 and genetic advance as percent of mean is of great importance to the breeder for making criteria for improvement in a complex character. With these considerations in mind, the present study focuses on assessment of the genetic variability, $\mathrm{h} 2$ and genetic advance for yield and yield component traits.

\section{Materials and Methods}

The present investigation was carried out at the Field Experimentation Centre, Department of Genetics and Plant Breeding, SHUATS, Prayagraj (U.P.) during Kharif, 2019. The University is situated on the left side of Prayagraj - Rewa National Highway, about 5 $\mathrm{km}$ away from Prayagraj city. All types of facilities necessary for cultivation of successful crop including field preparation, inputs and irrigation facilities were provided from the Department of Genetics and Plant 
Breeding, SHUATS, Prayagraj (U.P.). The experiment was conducted in randomized block design with 21 genotypes the genotypes were replicated 3 times. The row to row spacing was $30 \mathrm{~cm}$ and plant to plant distance was $10 \mathrm{~cm}$. The 5 competitive plants from each of the replication were tagged and observations were taken from these tagged plants at various stages of the crop plant growth. Data were recorded from 13 characters viz, days to $50 \%$ flowering, days to maturity, plant height $(\mathrm{cm})$, number of primary branches per plant, number of cluster per plant, number of pods per cluster, number of pods per plant, pod length $(\mathrm{cm})$, number of seeds per pod, 100 seed weight (g), biological yield (g), harvest index and grain yield per plant (g). Mean values were computed data were analyzed for analysis of variance as suggested by (Fisher, 1936) and coefficient of variances as well as heritability (in broad sense), as suggested by Burton and Devane (1953). The estimates of genetic advance were obtained by the formula suggested by Lush (1949) and Johnson et al., (1955).Phenotypic and genotypic correlation and path coefficients of variation were computed as per the method given by Dewey and Lu (1959).

\section{Results and Discussion}

The analysis of variance as a measure of variability indicated significant differences amongst the genotypes for all the characters, viz. days to $50 \%$ flowering, days to maturity, plant height $(\mathrm{cm})$, number of primary branches per plant, number of clusters per plant, number of pods per cluster, number of pods per plant, number of seeds per pod, pod length, 100seed weight (g), biological yield, harvest index and grain yield per plant. Rao et al., (2006), Singh et al., (2009), Reddy et al., (2011), Hemavathy et al., (2015) and Dhoot et al., (2017) also reported significant differences for all the characters studied. The presence of sufficient variability indicated that the materials of mungbean under study were good enough for further study. The phenotypic coefficient of variation (PCV) was found higher than that of genotypic coefficient of variation $(\mathrm{GCV})$ for all the traits, suggesting the role of environmental factors on various characters, also suggested by Khajudparn and Tantasawat (2011). The genotypes showed significant differences in respect of all the characters studied (Table 1). High PCV and GCV estimates were observed for number of primary branches per plant. Moderate GCV and PCV were found for 100 seed weight, number of clusters per plant and plant height. Similar results were also observed by Nand and Anuradha (2013) and Pandey et al., (2007) for number of branches per plant, Rao et al., (2007), Makeen et al., (2007) and Kumar and Choudhary (2007) for 100 seed weight. Lowest PCV and GCV estimates were obtained for days to $50 \%$ flowering and days to maturity. These results are in conformity with Makeen et al., (2007), Rao et al., (2007), Nand and Anuradha (2013) and Kumhar and Choudhary (2007). The results obtained for PCV and GCV showed that there is considerable possibility of further improvement through intermating followed by appropriate selection for these characters.

High genetic advance coupled with high heritability were observed for plant height, number of branches per plant, plant height, 100 -seed weight, number of clusters per plant. This suggested the preponderance of additive gene action with low environmental influence for the determination of these characters and could be effective in phenotypic selection.

Similar results were obtained by Hemavathy et al., (2015) Pandey et al., (2007) for plant height, Rao et al., (2007) for number of primary branches per plant, 100 seed weight, Hari Kesh et al., (2017) for number of branches per plant and Godakh et al., (2013) for 100 seed weight and number of branches 
per plant. The character, days to flowering $50 \%$ exhibited moderate heritability with low genetic advance may be due to non-additive gene action. Similar observations were reported in mungbean by Singh et al., (2009) for days to $50 \%$ flowering. Yield, a complex polygenic trait has a large number of other contributing component traits. Correlation analysis reveals the relationship of dependent variable yield with its independent variables, thus association of various traits would determine their relative significance to improve yield. Grain yield per plant exhibited negative correlation with days to flowering and days to maturity suggesting the importance of short duration genotypes. On the other hand grain yield per plant exhibited positive correlation with plant height, number of branches per plant, number of pods per cluster harvest index, number of pods per plant, pod length and number of seeds per pod indicating their importance in increasing yield. These results are in conformity with Hemavathy et al., (2015) for number of pods per plant and number of seeds per pod, Anand et al., (2016) for number of pods per plant, Baisakh et al., (2016) for number of pods per plant and number of seeds per pod, Makeen et al., (2007) for number of pods per plant and number of seeds per pod, Godakh et al.,(2013) and Dgefaet et al.,(2014)for harvest index, number of pods per plant, number of primary branches per plant and number of seeds per pod and Tiwari et al., (2014) for number of pods per plant and harvest index. Garget et al., (2017) for harvest index.

Table.1 Genetic parameters for 13 characters in mungbean

\begin{tabular}{|c|c|c|c|c|c|c|c|c|}
\hline $\begin{array}{l}\text { Sl. } \\
\text { No. }\end{array}$ & Character & VG & $\mathbf{V P}$ & $\begin{array}{l}\text { GCV } \\
(\%)\end{array}$ & $\begin{array}{r}\text { PCV } \\
(\%)\end{array}$ & $\begin{array}{c}\mathbf{h}^{2} \mathbf{b s} \\
\%\end{array}$ & GA & $\begin{array}{c}\text { GA as \% } \\
\text { mean }\end{array}$ \\
\hline 1 & $\begin{array}{c}\text { Days to } 50 \% \\
\text { Flowering }\end{array}$ & 1.11 & 1.98 & 2.89 & 3.87 & 55.93 & 1.62 & 4.45 \\
\hline 2 & Days to maturity & 2.54 & 9.30 & 2.86 & 5.48 & 27.31 & 1.72 & 3.08 \\
\hline 3 & Plant height & 146.80 & 184.50 & 13.56 & 15.20 & 79.57 & 22.26 & 24.91 \\
\hline 4 & $\begin{array}{c}\text { Primary Branches } \\
\text { Per Plant }\end{array}$ & 0.25 & 0.41 & 21.27 & 26.97 & 62.21 & 0.82 & 34.55 \\
\hline 5 & $\begin{array}{c}\text { No. Of } \\
\text { Clusters Per Plant }\end{array}$ & 0.21 & 0.26 & 14.48 & 16.31 & 78.79 & 0.84 & 26.47 \\
\hline 6 & $\begin{array}{l}\text { No. Of Pods Per } \\
\text { Cluster }\end{array}$ & 0.03 & 0.09 & 5.77 & 10.86 & 28.26 & 0.17 & 6.32 \\
\hline 7 & $\begin{array}{c}\text { No. Of Pods Per } \\
\text { Plant }\end{array}$ & 0.31 & 1.27 & 5.44 & 11.07 & 24.15 & 0.56 & 5.51 \\
\hline 8 & Pod Length & 0.04 & 0.06 & 2.97 & 3.94 & 56.85 & 0.29 & 4.61 \\
\hline 9 & $\begin{array}{c}\text { No. Of. Seeds Per } \\
\text { pod }\end{array}$ & 0.11 & 0.25 & 4.73 & 7.34 & 41.56 & 0.43 & 6.28 \\
\hline 10 & 100 Seed Weight & 0.31 & 0.36 & 16.72 & 18.08 & 85.60 & 1.06 & 31.87 \\
\hline 11 & Biological Yield & 0.32 & 0.97 & 3.19 & 5.54 & 33.19 & 0.67 & 3.79 \\
\hline 12 & Harvest Index & 10.16 & 15.97 & 9.19 & 11.51 & 63.79 & 5.26 & 15.12 \\
\hline 13 & $\begin{array}{c}\text { Grain Yield Per } \\
\text { Plant }\end{array}$ & 0.29 & 0.40 & 8.68 & 10.28 & 71.34 & 0.93 & 15.10 \\
\hline \multicolumn{9}{|c|}{$\mathrm{VP}=$ Phenotypic variance } \\
\hline
\end{tabular}


Table.2 Correlation coefficient between yield and its related traits in mungbean genotypes at genotypic level

\begin{tabular}{|c|c|c|c|c|c|c|c|c|c|c|c|c|c|}
\hline Sr.No & Character & $\begin{array}{l}\text { Days to } \\
\text { Maturity }\end{array}$ & $\begin{array}{c}\text { Plant } \\
\text { Height }\end{array}$ & $\begin{array}{l}\text { Primary } \\
\text { Branches } \\
\text { per Plant }\end{array}$ & $\begin{array}{c}\text { Clusters } \\
\text { per } \\
\text { plant }\end{array}$ & $\begin{array}{c}\text { Pods } \\
\text { per } \\
\text { cluster }\end{array}$ & $\begin{array}{c}\text { Pods } \\
\text { per } \\
\text { Plant }\end{array}$ & $\begin{array}{c}\text { Pod } \\
\text { Length }\end{array}$ & $\begin{array}{c}\text { Seeds } \\
\text { per } \\
\text { pod }\end{array}$ & $\begin{array}{c}100 \\
\text { Seed } \\
\text { Weight }\end{array}$ & $\begin{array}{l}\text { Biological } \\
\text { yield }\end{array}$ & $\begin{array}{l}\text { Harvest } \\
\text { Index }\end{array}$ & $\begin{array}{c}\text { Grain } \\
\text { Yield per } \\
\text { plant }\end{array}$ \\
\hline 1 & $\begin{array}{c}\text { Days to } 50 \% \\
\text { Flowering }\end{array}$ & $-0.512^{* *}$ & 0.088 & -0.199 & -0.242 & $-0.734^{* *}$ & $-0.245^{*}$ & -0.043 & 0.047 & 0.235 & $0.247^{*}$ & -0.133 & -0.064 \\
\hline 2 & $\begin{array}{l}\text { Days to } \\
\text { maturity }\end{array}$ & 1 & $0.364^{* *}$ & -0.208 & $0.775^{* *}$ & 0.233 & $1.202^{* *}$ & -0.057 & $1.102^{* *}$ & $0.259^{*}$ & $0.267^{*}$ & -0.182 & -0.106 \\
\hline 3 & Plant Height & & 1 & -0.234 & $-0.489^{* *}$ & -0.122 & $0.330^{* *}$ & $-0.300^{*}$ & $0.334^{* *}$ & 0.155 & 0.154 & 0.021 & 0.063 \\
\hline 4 & $\begin{array}{l}\text { Primary } \\
\text { Branches } \\
\text { per Plant }\end{array}$ & & & 1 & 0.174 & $0.673^{* *}$ & 0.084 & -0.109 & -0.025 & 0.163 & -0.096 & $0.493^{* *}$ & $0.488^{* *}$ \\
\hline 5 & $\begin{array}{c}\text { Clusters per } \\
\text { Plant }\end{array}$ & & & & 1 & 0.241 & $0.590^{* *}$ & 0.169 & $0.509^{* *}$ & 0.033 & 0.041 & -0.042 & -0.02 \\
\hline 6 & $\begin{array}{c}\text { Pods Per } \\
\text { Cluster }\end{array}$ & & & & & 1 & $0.458^{* *}$ & -0.191 & $0.773^{* *}$ & -0.067 & $-0.442^{* *}$ & $0.811^{* *}$ & $0.703^{* *}$ \\
\hline 7 & $\begin{array}{c}\text { Pods per } \\
\text { Plant }\end{array}$ & & & & & & 1 & $-0.432^{* *}$ & $1.110^{* *}$ & 0.154 & $0.447^{* *}$ & $-0.289^{*}$ & $0.528^{* *}$ \\
\hline 8 & Pod Length & & & & & & & 1 & $\begin{array}{c}- \\
0.285^{*}\end{array}$ & 0.123 & $-1.104^{* *}$ & 0.152 & $-0.251^{*}$ \\
\hline 9 & $\begin{array}{l}\text { Seeds per } \\
\text { pod }\end{array}$ & & & & & & & & 1 & 0.146 & 0.199 & $0.363^{\text {** }}$ & $0.436^{* *}$ \\
\hline 10 & $\begin{array}{l}100 \text { Seed } \\
\text { Weight }\end{array}$ & & & & & & & & & 1 & $-0.318^{* *}$ & $0.447^{* * *}$ & $0.351^{* *}$ \\
\hline 11 & $\begin{array}{l}\text { Biological } \\
\text { Yield }\end{array}$ & & & & & & & & & & 1 & $-0.314^{*}$ & 0.015 \\
\hline 12 & $\begin{array}{c}\text { Harvest } \\
\text { Index }\end{array}$ & & & & & & & & & & & 1 & $0.944^{* *}$ \\
\hline
\end{tabular}

$1 \% * *$ Level of significance $5 \% *$ Level of significance 
Table.3 Correlation coefficient between yield and its related traits in Mungbean genotypes at phenotypic level

\begin{tabular}{|c|c|c|c|c|c|c|c|c|c|c|c|c|c|}
\hline Sr.No & Character & $\begin{array}{l}\text { Days to } \\
\text { Maturity }\end{array}$ & $\begin{array}{c}\text { Plant } \\
\text { Height }\end{array}$ & $\begin{array}{l}\text { Primary } \\
\text { Branches } \\
\text { per Plant }\end{array}$ & $\begin{array}{c}\text { Clusters } \\
\text { per } \\
\text { plant }\end{array}$ & $\begin{array}{c}\text { Pods } \\
\text { per } \\
\text { cluster }\end{array}$ & $\begin{array}{l}\text { Pod per } \\
\text { Plant }\end{array}$ & $\begin{array}{c}\text { Pod } \\
\text { Length }\end{array}$ & $\begin{array}{c}\text { Seeds } \\
\text { per } \\
\text { pod }\end{array}$ & $\begin{array}{c}100 \\
\text { Seed } \\
\text { Weight }\end{array}$ & $\begin{array}{l}\text { Biological } \\
\text { yield }\end{array}$ & $\begin{array}{l}\text { Harvest } \\
\text { Index }\end{array}$ & $\begin{array}{c}\text { Grain } \\
\text { Yield per } \\
\text { plant }\end{array}$ \\
\hline 1 & $\begin{array}{c}\text { Days to } 50 \% \\
\text { Flowering }\end{array}$ & -0.206 & 0.013 & $-0.304^{*}$ & -0.137 & -0.136 & -0.116 & -0.107 & -0.131 & $0.353^{* *}$ & 0.137 & $-0.369^{* *}$ & -0.103 \\
\hline 2 & $\begin{array}{c}\text { Days to } \\
\text { maturity }\end{array}$ & 1 & 0.145 & 0.028 & $0.404^{* *}$ & 0.021 & $0.517^{* *}$ & -0.013 & $0.371^{* *}$ & 0.121 & $0.284^{*}$ & -0.022 & 0.019 \\
\hline 3 & Plant Height & & 1 & -0.202 & $-0.388^{* *}$ & -0.087 & 0.124 & $-0.297^{*}$ & 0.108 & 0.131 & 0.007 & $0.499^{* * *}$ & 0.107 \\
\hline 4 & $\begin{array}{l}\text { Primary } \\
\text { Branches } \\
\text { per Plant }\end{array}$ & & & 1 & 0.157 & $0.344^{* *}$ & 0.03 & $-0.288^{*}$ & 0.044 & 0.151 & 0.019 & $0.305^{*}$ & $0.355^{* * *}$ \\
\hline 5 & $\begin{array}{l}\text { Clusters per } \\
\text { Plant }\end{array}$ & & & & 1 & 0.112 & $0.329^{* *}$ & 0.138 & $0.312^{*}$ & 0.05 & $-0.343^{* *}$ & -0.054 & -0.08 \\
\hline 6 & $\begin{array}{l}\text { Pods per } \\
\text { Cluster }\end{array}$ & & & & & 1 & 0.113 & -0.111 & $0.302^{*}$ & $-0.291^{*}$ & -0.042 & $0.250^{*}$ & $0.255^{*}$ \\
\hline 7 & $\begin{array}{l}\text { Pods per } \\
\text { Plant }\end{array}$ & & & & & & 1 & -0.073 & $0.367^{* *}$ & 0.082 & 0.084 & $-0.598^{* *}$ & $0.455^{* *}$ \\
\hline 8 & Pod Length & & & & & & & 1 & $0.358^{* * *}$ & 0.027 & $-0.389^{* * *}$ & 0.046 & -0.161 \\
\hline 9 & $\begin{array}{l}\text { Seeds per } \\
\text { pod }\end{array}$ & & & & & & & & 1 & 0.092 & $0.414^{* *}$ & 0.229 & $0.309^{*}$ \\
\hline 10 & $\begin{array}{l}100 \text { Seed } \\
\text { Weight }\end{array}$ & & & & & & & & & 1 & -0.19 & $0.370^{* * *}$ & $0.306^{*}$ \\
\hline 11 & $\begin{array}{c}\text { Biological } \\
\text { Yield }\end{array}$ & & & & & & & & & & 1 & $-0.436^{* *}$ & 0.044 \\
\hline 12 & $\begin{array}{c}\text { Harvest } \\
\text { Index }\end{array}$ & & & & & & & & & & & 1 & $0.878^{* *}$ \\
\hline
\end{tabular}

$1 \% * *$ Level of significance $5 \%$ * Level of significance 
Table.4 Direct and indirect effects between yield and its different traits in Mungbean genotypes at genotypic level

\begin{tabular}{|c|c|c|c|c|c|c|c|c|c|c|c|c|c|c|}
\hline Sr.No & Character & $\begin{array}{c}\text { Days to } \\
\mathbf{5 0 \%} \\
\text { Flowering }\end{array}$ & $\begin{array}{l}\text { Days to } \\
\text { Maturity }\end{array}$ & $\begin{array}{c}\text { Plant } \\
\text { Height }\end{array}$ & $\begin{array}{l}\text { Primary } \\
\text { Branches } \\
\text { per Plant }\end{array}$ & $\begin{array}{c}\text { Clusters } \\
\text { per } \\
\text { plant }\end{array}$ & $\begin{array}{c}\text { Pod } \\
\text { per } \\
\text { cluster }\end{array}$ & $\begin{array}{l}\text { Pods } \\
\text { Per } \\
\text { Plant }\end{array}$ & $\begin{array}{c}\text { Pod } \\
\text { Length }\end{array}$ & $\begin{array}{c}\text { Seeds } \\
\text { per } \\
\text { pod }\end{array}$ & $\begin{array}{c}100 \\
\text { Seed } \\
\text { Weight }\end{array}$ & $\begin{array}{l}\text { Biological } \\
\text { yield }\end{array}$ & $\begin{array}{c}\text { Harvest } \\
\text { Index }\end{array}$ & $\begin{array}{c}\text { Grain } \\
\text { Yield per } \\
\text { plant }\end{array}$ \\
\hline 1 & $\begin{array}{c}\text { Days to } \\
50 \% \\
\text { Flowering }\end{array}$ & -0.0498 & -0.0385 & $\begin{array}{c}- \\
0.0117\end{array}$ & -0.0108 & 0.0485 & 0.0457 & -0.0133 & -0.0066 & 0.0017 & -0.0034 & 0.1224 & -0.1479 & -0.057 \\
\hline 2 & $\begin{array}{l}\text { Days to } \\
\text { maturity }\end{array}$ & 0.0255 & 0.0752 & $\begin{array}{c}- \\
0.0488\end{array}$ & -0.0112 & -0.1554 & $0 . \overline{-}$ & 0.0653 & -0.0087 & 0.0399 & -0.0037 & 0.1323 & -0.2019 & -0.256 \\
\hline 3 & $\begin{array}{c}\text { Plant } \\
\text { Height }\end{array}$ & -0.0044 & 0.0274 & 0.1340 & -0.0127 & 0.0979 & 0.0076 & 0.0180 & -0.0459 & 0.0121 & -0.0022 & 0.0765 & 0.0229 & -0.202 \\
\hline 4 & $\begin{array}{l}\text { Primary } \\
\text { Branches } \\
\text { per Plant }\end{array}$ & 0.0099 & -0.0157 & 0.0314 & 0.0539 & -0.0349 & $0 . \overline{0419}$ & 0.0046 & -0.0167 & -0.0009 & -0.0023 & -0.0474 & 0.5478 & -0.127 \\
\hline 5 & $\begin{array}{l}\text { Clusters } \\
\text { per Plant }\end{array}$ & 0.0121 & 0.0583 & 0.0655 & 0.0094 & -0.2003 & $0 . \overline{0}$ & 0.0321 & 0.0259 & 0.0184 & -0.0005 & 0.0204 & -0.0467 & -0.074 \\
\hline 6 & $\begin{array}{l}\text { Pods per } \\
\text { Cluster }\end{array}$ & 0.0366 & 0.0175 & 0.0164 & 0.0363 & -0.0483 & $\begin{array}{c}- \\
0.0622\end{array}$ & 0.0249 & -0.0292 & 0.0280 & 0.0010 & -0.2188 & 0.9005 & 0.334 \\
\hline 7 & $\begin{array}{l}\text { Pods per } \\
\text { Plant }\end{array}$ & 0.0122 & 0.0904 & $\begin{array}{c}- \\
0.0442\end{array}$ & 0.0045 & -0.1181 & $\begin{array}{c}- \\
0.0285\end{array}$ & 0.0544 & -0.0662 & 0.0402 & -0.0022 & 0.2216 & -0.3212 & 0.581 \\
\hline 8 & $\begin{array}{c}\text { Pod } \\
\text { Length }\end{array}$ & 0.0021 & -0.0043 & 0.0402 & -0.0059 & -0.0339 & 0.0119 & -0.0235 & 0.1531 & -0.0103 & -0.0018 & -0.5466 & 0.1683 & 0.315 \\
\hline 9 & $\begin{array}{l}\text { Seeds per } \\
\text { pod }\end{array}$ & -0.0023 & 0.0829 & $\begin{array}{c}- \\
0.0447\end{array}$ & -0.0014 & -0.1019 & $\begin{array}{c}- \\
0.0481\end{array}$ & 0.0604 & -0.0436 & 0.0362 & -0.0021 & 0.0983 & 0.4028 & -0.382 \\
\hline 10 & $\begin{array}{l}100 \text { Seed } \\
\text { Weight }\end{array}$ & -0.0117 & 0.0195 & $\begin{array}{c}- \\
0.0208\end{array}$ & 0.0088 & -0.0065 & 0.0042 & 0.0084 & 0.0188 & 0.0053 & -0.0144 & -0.1574 & 0.4970 & -0.240 \\
\hline 11 & $\begin{array}{l}\text { Biological } \\
\text { Yield }\end{array}$ & -0.0123 & 0.0201 & $\begin{array}{c}- \\
0.0207\end{array}$ & -0.0052 & -0.0083 & 0.0275 & 0.0243 & -0.1690 & 0.0072 & 0.0046 & 0.4952 & -0.3484 & -0.263 \\
\hline 12 & $\begin{array}{c}\text { Harvest } \\
\text { Index }\end{array}$ & 0.0066 & -0.0137 & $\begin{array}{c}- \\
0.0028\end{array}$ & 0.0266 & 0.0084 & $\begin{array}{c}- \\
0.0504\end{array}$ & -0.0157 & 0.0232 & 0.0131 & -0.0065 & -0.1553 & 0.1107 & 0.839 \\
\hline
\end{tabular}

Residual are 0.00629 
Table.5 Direct and indirect effects between yield and its different traits in Mungbean genotypes at phenotypic level

\begin{tabular}{|c|c|c|c|c|c|c|c|c|c|c|c|c|c|c|}
\hline $\begin{array}{l}\text { Sr. } \\
\text { No }\end{array}$ & Character & $\begin{array}{c}\text { Days to } \\
50 \% \\
\text { Flowering }\end{array}$ & $\begin{array}{l}\text { Days to } \\
\text { Maturity }\end{array}$ & $\begin{array}{l}\text { Plant } \\
\text { Height }\end{array}$ & $\begin{array}{l}\text { Primary } \\
\text { Branches } \\
\text { per Plant }\end{array}$ & $\begin{array}{c}\text { Clusters } \\
\text { per } \\
\text { plant }\end{array}$ & $\begin{array}{c}\text { Pods } \\
\text { per } \\
\text { cluster }\end{array}$ & $\begin{array}{c}\text { Pods } \\
\text { per } \\
\text { Plant }\end{array}$ & $\begin{array}{c}\text { Pod } \\
\text { Length }\end{array}$ & $\begin{array}{c}\text { Seeds } \\
\text { per } \\
\text { pod }\end{array}$ & $\begin{array}{c}100 \\
\text { Seed } \\
\text { Weight }\end{array}$ & $\begin{array}{l}\text { Biological } \\
\text { yield }\end{array}$ & $\begin{array}{c}\text { Harvest } \\
\text { Index }\end{array}$ & $\begin{array}{c}\text { Grain } \\
\text { Yield per } \\
\text { plant }\end{array}$ \\
\hline 1 & $\begin{array}{c}\text { Days to } \\
\mathbf{5 0 \%} \\
\text { Flowering }\end{array}$ & -0.0100 & -0.0014 & 0.0000 & -0.0010 & -0.0008 & 0.0001 & 0.0023 & 0.0012 & 0.0004 & -0.0159 & 0.0719 & -0.1654 & 0.351 \\
\hline 2 & $\begin{array}{l}\text { Days to } \\
\text { maturity }\end{array}$ & 0.0021 & 0.0069 & $\begin{array}{c}- \\
0.0005\end{array}$ & 0.0001 & 0.0023 & 0.0000 & -0.0104 & 0.0001 & -0.0010 & -0.0563 & 0.0440 & -0.0244 & -0.135 \\
\hline 3 & $\begin{array}{c}\text { Plant } \\
\text { Height }\end{array}$ & -0.0001 & 0.0010 & $\stackrel{-}{0.0034}$ & -0.0010 & -0.0022 & 0.0001 & -0.0025 & 0.0025 & -0.0003 & -0.0053 & 0.0039 & 0.1094 & -0.047 \\
\hline 4 & $\begin{array}{l}\text { Primary } \\
\text { Branches } \\
\text { per Plant }\end{array}$ & 0.0020 & 0.0002 & 0.0007 & 0.0048 & 0.0009 & $\begin{array}{c}- \\
0.0003\end{array}$ & -0.0006 & 0.0013 & -0.0001 & -0.0009 & 0.0098 & 0.3363 & -0.323 \\
\hline 5 & $\begin{array}{l}\text { Clusters } \\
\text { per Plant }\end{array}$ & 0.0014 & 0.0028 & 0.0013 & 0.0008 & 0.0056 & $\begin{array}{c}- \\
0.0001 \\
\end{array}$ & -0.0066 & -0.0015 & -0.0009 & -0.0026 & -0.0224 & -0.0599 & -0.031 \\
\hline 6 & $\begin{array}{l}\text { Pods per } \\
\text { Cluster }\end{array}$ & 0.0014 & 0.0001 & 0.0003 & 0.0017 & 0.0006 & $\stackrel{-}{0.0010}$ & -0.0023 & 0.0012 & -0.0008 & -0.0057 & -0.0219 & 0.2756 & 0.371 \\
\hline 7 & $\begin{array}{c}\text { Pods per } \\
\text { Plant }\end{array}$ & 0.0012 & 0.0036 & $\begin{array}{c}- \\
0.0004\end{array}$ & 0.0001 & 0.0019 & $\begin{array}{c}- \\
0.0001\end{array}$ & -0.0200 & 0.0008 & -0.0010 & 0.0025 & 0.0443 & -0.1083 & 0.485 \\
\hline 8 & $\begin{array}{c}\text { Pod } \\
\text { Length }\end{array}$ & 0.0011 & -0.0001 & 0.0008 & -0.0006 & 0.0008 & 0.0001 & 0.0015 & -0.0111 & 0.0004 & 0.0095 & -0.2040 & 0.0506 & -0.146 \\
\hline 9 & $\begin{array}{l}\text { Seeds per } \\
\text { pod }\end{array}$ & 0.0013 & 0.0026 & $\begin{array}{c}- \\
0.0003 \\
\end{array}$ & 0.0002 & 0.0018 & 0.0003 & -0.0074 & 0.0018 & -0.0028 & -0.0257 & 0.0597 & 0.2524 & 0.281 \\
\hline 10 & $\begin{array}{c}100 \text { Seed } \\
\text { Weight }\end{array}$ & -0.0015 & 0.0008 & $\begin{array}{c}- \\
0.0004\end{array}$ & 0.0007 & 0.0003 & 0.0001 & -0.0017 & -0.0003 & -0.0003 & -0.0254 & -0.0997 & 0.4084 & -0.141 \\
\hline 11 & $\begin{array}{l}\text { Biological } \\
\text { Yield }\end{array}$ & -0.0014 & 0.0006 & 0.0000 & 0.0001 & -0.0002 & 0.0000 & -0.0017 & 0.0043 & -0.0003 & 0.0199 & 0.5248 & -0.4818 & 0.018 \\
\hline 12 & $\begin{array}{c}\text { Harvest } \\
\text { Index }\end{array}$ & 0.0015 & -0.0002 & $\begin{array}{c}- \\
0.0003\end{array}$ & 0.0015 & -0.0003 & $\begin{array}{c}- \\
0.0003\end{array}$ & 0.0020 & -0.0005 & -0.0006 & -0.0365 & -0.2290 & 0.1040 & 0.785 \\
\hline
\end{tabular}

Residual are $\mathbf{0 . 0 0 3 4}$ 
All the direct effects were less than one which indicates that influences due to multicollinearity were minimal. The critical evaluation of path coefficient for agronomic traits, indicate that biological yield had the highest direct and positive effect on grain yield per plant, followed by harvest index, days to maturity and number of primary branches per plant. Similar results were also reported by Rao et al., (2006) for harvest index, days to maturity, biological yield, Pandey et al., (2007) for harvest index, biological yield, Gadhak et al., (2013) for harvest index, biological yield, number of primary branches per plant and Tiwari et al., (2014) for harvest index, Arshad et al., (2009) for number primary branches per plant, Sreethy et al., (2017)for biological yield. So far from the combined results of correlation coefficient and path analysis, it may be concluded that days to maturity, number of primary branches per plant, biological yield and harvest index are major components of grain yield and should be given top priority while formulating a selection strategy for improvement of yield in mungbean.

\section{Acknowledgement}

We are thankful to the Honorable Vice Chancellor, HOD, Teaching and non-Teaching staff of the Department of Genetics and Plant Breeding, Naini Agricultural Institute, Sam Higginbottom University of Agriculture, Technology and Sciences, Prayagraj-211007, U.P., for providing all necessary facilities and support.

\section{References}

Anand, G., Anandhi, K. and Paulpandi, V. K. (2016). Genetic variability, correlation and path analysis for yield and yield components in F6 families of Greengram (Vigna radiata (L.) Wilczek) under rainfed condition.
Electronic Journal Plant Breeding, 7:434-437.

Anuradha, N., Patro, T. S. and Kumar Raj, S. (2019). Study on genetic variability, heritability and correlation analysis for grain yield and yield attributing traits in green gram [Vigna radiata (L.) Wilczek.]. International Journal of Chemical Studies, 7(3): 2050-2052.

Baisakh, B., Swain, S. C., Panigrahi, K. K., Das, T. R. and Mohanty, A. (2016). Estimation of Genetic Variability and Character Association in Micro Mutant Lines of Greengram [Vigna radiata (L.)Wilczek] for Yield Attributes and Cold Tolerance. Legume Genomics Genet., 7:1-9.

Burton, G. W. (1952). Quantitative inheritance in grasses proceedings. International Grassland Congress, 1: 227-283.

De Candolle, A. (1886). Origin of cultivated plants. Hafner Publication Co., New York, N. Y. (Reprint of $2^{\text {nd }}$ ed. 1959).

Degefa, I., Petros, Y. and Andargie, M. (2014). Genetic variability, heritability and genetic advance in Mung bean (Vigna radiata L. Wilczek) accessions. Plant Science Today, 1(2).

Dewey, D. R. and Lu, K. H. (1959). Correlation and path coefficient analysis of crested wheatgrass seed production. Agronomic Journal, 5(1): 515518

Dhoot, R., Modha, K. G., Kumar, D. and Dhoot, M. (2017).Correlations and Path Analysis Studies on Yield and its Components in Mungbean (Vigna radiata (L.) Wilczek). International journal Current Microbiology Applied Science, 6(5): 370-378.

Gadakh, S. S., Dethe, A. M. and Kathale, M. N. (2013). Genetic variability, correlations and path analysis studies on yield and its components in Mungbean (Vigna radiata (L.) Wilczek). Bioinfolet, 10(2A): 441-447. 
Hemavathy, A. T., Shunmugavalli, N. and Anand, G. (2015). Genetic variability, correlation and path co-efficient studies on yield and its components in mungbean [Vigna radiata (L.)Wilezek]. Legume Resources 38 (4): 442-446.

Khajudparn, P. and Tantasawat, P. (2011). Relationships and variability of agronomic and physiological characters in mungbean. African Journal or Biotechnology 10(49): 9992-10000.

Kumhar, S. R. and Chaudhary, B. R. (2007). Genetic diversity and variability in Mungbean [Vigna radiata (L.)Wilczek]. Journal Plant Genetics Resources, 20(2): 203-208.

Makeen, K., Abrahim, G., Jan, A. and Singh, A. K. (2007). Genetic variability and correlations studies on yield and its components in mungbean (Vigna radiata (L.) Wilezek). Journal Agronomy 6(1): 216-218.

Makeen, K., Garard, A. Arif J. and Archana K. S. (2007) Genetic variability and correlation studies on yield and its components in mungbean (Vigna radiata (L). Wilckez). Journal Agronomy 6: 216-218.

Pandey, M. K., Srivastava, N. and Kole, C. R. (2007).Selection strategy for augmentation of seed yield in mungbean (Vigna radiate (L.) Wilczek). Legume Resources, 30(4): 243-249.

Rao, C. M., Rao, Y. K. and Reddy, M. (2006). Genetic variability and path analysis in Mungbean. Legume Resource, 29(3): 216-218.

Singh, A., Singh, S. K., Sirohi, A. and Yadav, R. (2009). Genetic variability and correlation studies in green gram (Vigna radiata (L.) Wilczek). Progressive agriculture, 9(1): 59-62.

Tiwari, A., Mishra, A. K. and Nag, S. K. (2014). Correlation and path coefficient analysis for seed yield and its components in Mungbean (Vigna radiata (L.) Wilczek). Trends Bioscience 7(1): 42-45.

\section{How to cite this article:}

Gitesh Padol, G. R. Lavanya and Rohini Kurandale. 2021. Estimation of Character Association among Yield Attributing Traits in Mungbean (Vigna radiata (L.) Wilczek). Int.J.Curr.Microbiol.App.Sci. 10(09): 183-192. doi: https://doi.org/10.20546/ijcmas.2021.1009.021 